\title{
Perfil dos Bolsistas de Produtividade em Pesquisa do CNPQ em Psicologia
}

\author{
A profile of researchers in psychology with productivity \\ grants from Brazil's national council of technologiocal \\ and scientific development (CNPQ)
}

Perfil de los becarios de productividad en investigación del CNPQ en psicología

Guilherme Welter Wendt \& Carolina Saraiva de Macedo Lisboa

Pontifícia Universidade Católica do Rio Grande do Sul

Diogo Araújo DeSousa \& Sílvia Helena Koller Universidade Federal do Rio Grande do Sul
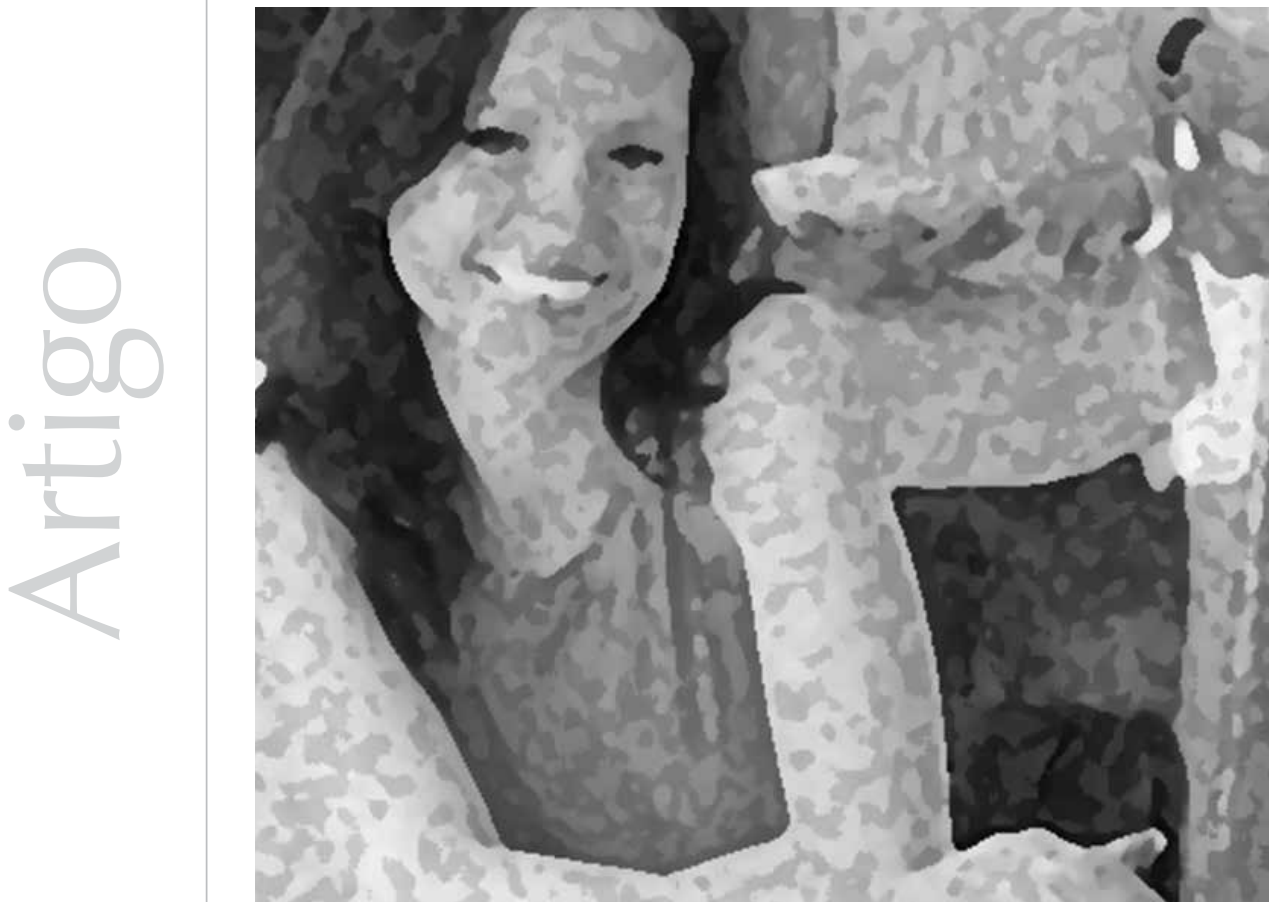
Resumo: Este estudo teve por objetivo analisar o perfil dos bolsistas de produtividade em pesquisa do $\mathrm{CNPq}$ da área de Psicologia segundo os dados declarados na Plataforma Lattes. O número de artigos, livros e capítulos de livro, bem como orientações de iniciação científica, mestrado, doutorado e pósdoutorado de 297 pesquisadores bolsistas da área foram coletados no decorrer da última semana de abril de 2011. Também foram coletados dados acerca das instituições às quais os bolsistas estão vinculados. Foram realizadas análises estatísticas descritivas (levantamento de frequências, médias e desvios-padrão) para caracterização da produtividade da amostra, e inferenciais (qui-quadrado e ANOVA) para comparar os bolsistas de cada categoria em relação ao número de publicações e de orientações e de dados demográficos e institucionais. Os resultados mostram que houve predomínio do sexo feminino (63\%) e da bolsa tipo $2(58,6 \%)$ entre os bolsistas. A maioria dos bolsistas está vinculada atualmente a instituições públicas (79,1\%). A Região Sudeste apresentou o maior número de bolsistas (53,6\%), seguida da Região Sul (18,5\%). Pesquisadores com bolsas de categorias mais altas (1A e 1B) apresentaram médias significativamente maiores quanto à produção de artigos, livros e capítulos de livro bem como ao número de orientações concluídas. As implicações e as limitações do estudo são discutidas.

Palavras-chave: Pesquisa científica. Cientistas. Sistema de créditos e Avaliação de pesquisadores. Atividades científicas e tecnológicas. Psicologia.

Abstract: The present work aimed to analyze the profile of researchers in psychology with productivity grants from Brazil's National Council of Technological and Scientific Development (CNPq), according to the information collected at the website called Lattes Platform. Numbers of papers, book chapters and books, as well as undergraduate, master and doctoral students' orientations were raised considering 297 researchers with productivity grants. This survey was conducted in the last week of April 2011. Information from the institutions where the researchers work were also collected. Descriptive analyses were held (frequencies, means and standard deviation of the study variables) to characterize the level of productivity of the sample, and inferential analyses were also conducted (Chi-square and ANOVA) to compare the productivity grants researchers of each category in what refers to the number of publications, orientations, social demographical data and institutional characteristics. The results showed the female gender (63\%) and the grant type $2(58,6 \%)$ were majority among productivity grants researchers. The majority of researchers works at public institutions $(79,1 \%)$. The Southeast showed the major number of productivity researchers $(53,6 \%)$, followed by the South (18,5\%). Researchers with grants type 1A e 1B, which means high categories, presented significant higher means of papers, books and book chapters publications, as well as finished students' orientations. The results implications and the study limitations are discussed.

Keywords: Scientific research. Scientists. Research policy evaluation. Productivity grants. Scientific and technical activities. Psychology.

Resumen: Este estudio tuvo como objetivo analizar el perfil de los becarios de productividad en investigación del CNPq del área de psicología según los datos declarados en la Plataforma Lattes. El número de artículos, libros y capítulos de libro, así como orientaciones de iniciación científica, maestría, doctorado y pos doctorado de 297 investigadores becarios del área fueron recolectados en el transcurso de la última semana de abril de 2011. También fueron recolectados datos acerca de las instituciones a las cuales los becarios están vinculados. Fueron realizados análisis estadísticos descriptivos (relevamiento de frecuencias, promedios y desviación estándar) para caracterización de la productividad de la muestra, e inferenciales (Ji-cuadrado y ANOVA) para comparar los becarios de cada categoría en relación al número de publicaciones y de orientaciones y de datos demográficos e institucionales. Los resultados muestran que hubo predominio del sexo femenino (63\%) y de la beca tipo 2 (58,6\%) entre los becarios. La mayoría de los becarios están vinculados actualmente a instituciones públicas (79,1\%). La Región Sudeste presentó el mayor número de becarios (53,6\%), seguida de la Región Sur (18,5\%). Investigadores con becas de categorías más altas $(1 \mathrm{~A}$ y $1 \mathrm{~B})$ presentaron promedios significativamente mayores en relación a la producción de artículos, libros y capítulos de libro así como al número de orientaciones concluidas. Las implicaciones y las limitaciones del estudio son discutidas.

Palabras claves: investigación científica. Cientistas. Evaluación de políticas de investigación. Actividades científicas e tecnológicas. Psicología. 
As universidades estão cada vez mais interessadas em avaliar os desempenhos dos seus docentes, tanto em termos de satisfação dos alunos com a sua atuação como também no que tange ao desempenho em pesquisa (Richard, et al., 2009; Teodorescu, 2000).
As taxas de produção acadêmica são utilizadas internacionalmente como um indicador de desempenho individual e institucional de acadêmicos (Creamer, 1998; Ramsden, 1994; Salgado \& Páez, 2007). A literatura atual sobre avaliação da produção de pesquisadores emprega geralmente critérios unilaterais, como as apreciações de seu trabalho e a produção por meio de avaliação por pares (Witte \& Rogge, 2010) e também pelo número de citações recebidas (Lee \& Bozeman, 2005).

As universidades estão cada vez mais interessadas em avaliar os desempenhos dos seus docentes, tanto em termos de satisfação dos alunos com a sua atuação como também no que tange ao desempenho em pesquisa (Richard et al., 2009; Teodorescu, 2000). As agências de fomento também avaliam o desempenho de pesquisadores e de programas de pós-graduação (pela produtividade de seus docentes). Alguns autores defendem uma forma de investigação do desempenho dos cientistas mais ampla, sugerindo o uso de instrumentos multidimensionais que considerem, além de aspectos relativos à experiência dos pesquisadores, aspectos demográficos, políticos e institucionais, bem como fatores pessoais, como motivação para pesquisar, sentimentos de autoeficácia diante da pesquisa e estabelecimento de objetivos (e.g., Avital \& Collopy, 2001).

No Brasil, a vários pesquisadores que mantêm maior (artigos publicados, livros, capítulos) e mais qualificada (em periódicos científicos internacionais e nacionais) produção acadêmica e que apresentam propostas de qualidade ao Conselho Nacional de Desenvolvimento Científico e Tecnológico (CNPq), tem sido concedida uma bolsa de Produtividade em Pesquisa (PQ). As bolsas PQ seguem uma hierarquia de três níveis. No mais alto grau, há as bolsas PQ Sênior, seguidas pelas bolsas PQ-1 e, por último, as bolsas PQ-2 e PQ-2F. As bolsas PQ-1 ainda se subdividem em 1A, 1B, 1C e 1D. A bolsa PQ Sênior tem caráter vitalício e pode ser concedida pelo Conselho Deliberativo do $\mathrm{CNPq}$ a pedido dos pesquisadores, caso estes tenham se mantido como bolsistas PQ 1A ou 1B por no mínimo 15 anos seguidos. As bolsas PQ-1 são concedidas, em princípio, aos docentes vinculados aos programas de pós-graduação, cuja experiência acadêmica já tenha resultado em produção significativa, bem como na orientação concluída de Mestres e Doutores. Variando em categorias, essas bolsas fornecem um subsídio financeiro mensal, e aqueles que são classificados como categoria 1 recebem um adicional grant para cobrir parte dos custos com pesquisa. Os pesquisadores com produtividade na categoria 2 não recebem esse adicional. Como explicam Santos, Cândido e Kuppens (2010), a bolsa PQ foi concebida como um incentivo aos pesquisadores com destacada produção científica para valorização de seu trabalho e dedicação frente a seus pares. No entanto, Emden (1998) ponderou que o auxílio financeiro, isoladamente, não configuraria um fator de garantia da produtividade desses pesquisadores. Assim, pode ser compreendido que o esforço em estabelecer políticas adicionais - como as adotadas em termos de bolsas de produtividade, prêmios e grants auxilia na efetiva manutenção da produção elevada dos cientistas.

No País, 14.616 bolsas de Produtividade em Pesquisa encontram-se atualmente em vigência (CNPq, 2012). As áreas que contam com maiores percentuais de bolsistas são: Física (6,23\%), Agronomia (5,53\%), Química (4,99\%), Medicina (3,58\%), Geociências $(3,09 \%)$, Ciências da Computação $(2,78 \%)$, Educação (2,61\%), Engenharia de Materiais e Metalúrgica (2,41\%), Engenharia Elétrica $(2,28 \%)$, Engenharia Mecânica $(2,11 \%)$, Matemática (2,07\%) e, em 12 o lugar, Psicologia $(2,03 \%)$. Estudos nacionais recentes buscaram apresentar o perfil dos pesquisadores bolsistas de produtividade em pesquisa, com o intuito 
de fornecer informações para o delineamento de políticas de desenvolvimento científico e tecnológico, a exemplo das áreas de Medicina (Mendes, Martelli, Souza, Qurino Filho, \& Martelli-Júnior, 2010), Química (Santos, Cândido, \& Kuppens, 2010), Odontologia (Cavalcanti \& Pereira, 2008) e saúde coletiva (Barata \& Goldbaum, 2003). No entanto, não foram encontrados trabalhos em Psicologia que atendessem, especificamente, a esse propósito. O trabalho de Hutz, Rocha, Spink e Menandro (2010) traz um panorama da produção intelectual dos programas de pósgraduação em Psicologia no Brasil, mas não especifica a produtividade dos bolsistas $\mathrm{CNPq}$ nos programas.

Assim, este estudo teve por objetivo analisar o perfil dos pesquisadores com bolsa de produtividade em pesquisa do CNPq na área da Psicologia. Dada a crescente demanda social, a presente investigação acerca do desempenho científico de pesquisadores será capaz de apresentar um cenário relativo às bolsas PQ na área da Psicologia. Tal retomada é relevante para a comunidade científica em geral no intuito de reavaliar metas acadêmicas e critérios de avaliação.

Adicionalmente, buscou-se relacionar o desempenho individual em pesquisa com características como a orientação de trabalhos de iniciação científica, mestrado, doutorado e pós-doutorado bem como analisar e comparar os resultados de acordo com variáveis como o sexo, localização geográfica e categoria administrativa da instituição à qual estão vinculados os pesquisadores. Por fim, são discutidos e sintetizados os dados em relação com os níveis da bolsa $\mathrm{PQ}$ do docente.

\section{Método}

Este estudo utilizou um delineamento descritivo transversal. Foi elaborada uma planilha com os dados de produtividade dos
297 pesquisadores com bolsas PQ da área de Psicologia. A relação dos bolsistas ativos foi obtida através do sítio do CNPq < www.cnpq. $\mathrm{br}>$. Os dados foram coletados do Sistema Eletrônico de Currículos da Plataforma Lattes, na última semana do mês de abril de 2011, a partir dos currículos de cada pesquisador(a). Foi utilizada essa plataforma, pois os pesquisadores, ao aceitarem a publicação do conteúdo dos seus currículos em domínio público, comprometem-se e responsabilizam-se pela veracidade das informações. A análise da consistência dessas informações não foi objeto de atenção deste trabalho.

Os dados coletados dos Currículos Lattes referentes a cada pesquisador(a) foram: sexo, instituição a que está vinculado, categoria administrativa (se federal, estadual ou privada) e Região do País, nível da bolsa PQ, publicações quanto a número de artigos, livros e capítulos de livros e número de orientações concluídas de iniciação científica, mestrado, doutorado e pós-doutorado. O número de publicações computado referiu-se ao período de 2007 a 2011, e o número de orientações concluídas a todo o período de atividade do docente, em consonância com os critérios frequentemente considerados quando do encaminhamento de pedidos de bolsas PQ (conforme CNPq, 2009). A escolha desses indicadores esteve em sintonia com os critérios adotados atualmente na recomendação para concessão de bolsas de produtividade. A seleção dos aspectos baseou-se nos critérios de avaliação da área de produção científica em Psicologia, a qual tem considerado que os itens de produção mais relevantes para a avaliação de produtividade são: artigos, capítulos, livros (os três ponderados a partir do Qualis), teses e dissertações e trabalhos completos em anais de eventos (com menor peso - quantitativo) (Hutz, Rocha, Spink \& Menandro, 2010).

Foram realizadas análises estatísticas descritivas (levantamento de frequências, 
médias e desvios-padrão) para caracterização da produtividade da amostra, e inferenciais (quiquadrado e ANOVA) para comparar os bolsistas de cada categoria em relação ao número de publicações e orientações, dados demográficos e institucionais.

\section{Resultados e discussão}

Os 297 pesquisadores analisados neste estudo foram provenientes de 49 instituições de ensino superior (IES) no Brasil. A Tabela 1 apresenta os dados relativos às instituições com um ranking das 11 instituições com maior número de bolsistas PQs em seus quadros. A Universidade de São Paulo apresentou o maior número de pesquisadores $(n=49 ; 16,5 \%)$, seguida da Universidade de Brasília $(n=28 ; 9,4 \%)$ e da Universidade Federal do Rio Grande do Sul ( $n=23 ; 7,7 \%)$.

Tabela 1. Ranking das instituições com maior número de bolsistas PQ por nível da bolsa

\begin{tabular}{|c|c|c|c|c|c|c|c|c|c|}
\hline \multirow[b]{2}{*}{ Instituiçãao } & \multirow[b]{2}{*}{ Número de Bolsistas } & \multirow[b]{2}{*}{$\%$ de Bolsas } & \multicolumn{7}{|c|}{ Nível da Bolsa } \\
\hline & & & $1 \mathrm{~A}$ & 1B & $1 \mathrm{C}$ & $1 \mathrm{D}$ & 2 & $2 \mathrm{~F}$ & SR \\
\hline USP & 49 & 16,5 & 4 & 9 & 4 & 8 & 23 & -- & 1 \\
\hline UNB & 28 & 9,4 & 1 & -- & 2 & 3 & 21 & 1 & -- \\
\hline UFRGS & 23 & 7,7 & 5 & 3 & 3 & 2 & 10 & -- & -- \\
\hline UFRJ & 15 & 5,1 & 1 & 1 & 1 & 2 & 10 & -- & -- \\
\hline UFSC & 13 & 4,4 & -- & 2 & 1 & -- & 10 & -- & -- \\
\hline UFPA & 12 & 4,0 & -- & 1 & 1 & 1 & 9 & -- & -- \\
\hline UERJ & 11 & 3,7 & 1 & -- & 2 & 2 & 6 & -- & -- \\
\hline UFRN & 10 & 3,4 & 1 & 2 & -- & 3 & 3 & 1 & -- \\
\hline PUC-Rio & 09 & 3,0 & 1 & 1 & 1 & 2 & 4 & -- & -- \\
\hline UFPB & 09 & 3,0 & -- & 1 & 1 & 1 & 6 & -- & -- \\
\hline UFMG & 09 & 3,0 & -- & 1 & 1 & 1 & 6 & -- & -- \\
\hline
\end{tabular}

A Tabela 2 mostra a comparação entre o número de bolsas PQ por Região do País e a proporção de bolsas por milhões de habitantes em cada Região e em todo o Brasil. Os dados populacionais utilizados referem-se ao último censo realizado pelo Instituto Brasileiro de Geografia e Estatística (IBGE, 2010). A Região Sudeste apresentou o maior número de bolsas de produtividade (53,9\%), enquanto os menores índices foram apresentados pelas Regiões Norte (4,4\%) e Nordeste (12,5\%). Já quando considerado o número de bolsas por milhão de habitantes, a Região Centro-oeste demonstrou a maior concentração de bolsas, seguida pela Região Sul.

Tabela 2. Distribuição das bolsas PQ pelas Regiões do Brasil

\begin{tabular}{ccccc}
\hline Região & Bolsas PQ (n) & Bolsas PQ (\%) & População & Bolsas PQ/milhão de habitantes \\
\hline Norte & 13 & 4,4 & 15.864 .454 & 0,819 \\
Nordeste & 37 & 12,5 & 53.081 .950 & 0,697 \\
Centro-oeste & 32 & 10,8 & 14.058 .094 & 2,276 \\
Sudeste & 160 & 53,9 & 80.364 .410 & 1,991 \\
Sul & 55 & 18,5 & 27.386 .891 & 2,008 \\
Brasil & 297 & 100 & 190.755 .799 & 1,557 \\
& & & & \\
\hline
\end{tabular}


A Tabela 3 resume outros dados relacionados à caracterização dos pesquisadores com bolsas PQ. A maioria dos bolsistas é do sexo feminino (63\%), o que contraria os achados de estudos com pesquisadores de outras áreas no Brasil, como Medicina (Mendes et al., 2010) e Química (Santos, Cândido, \& Kuppens, 2010), bem como os achados de Sax, Hagedorn, Arredondo e Dicrisi (2002) com uma amostra norte-americana representativa de professores universitários de todas as áreas. No estudo de Santos, Cândido e Kuppens (2010), que analisou os currículos dos 604 bolsistas de produtividade em pesquisa na área da Química do CNPq, 67,2\% eram do sexo masculino. Já no estudo norte-americano de Sax, et al as mulheres apresentaram desvantagem em relação aos pesquisadores de mesmo nível hierárquico do sexo masculino nos níveis de produção científica. Embora essa disparidade observada esteja decrescendo, são descritas hipóteses que possivelmente sejam válidas nos EUA e aqui no Brasil, com relação às taxas de produção acadêmica das mulheres, uma vez que, além das atividades profissionais, continuam sendo responsáveis principais pelo "cuidado com os filhos e tempo gasto em atividades domésticas" (Sax et al., 2002, p. 436). De acordo com Castro e Yamamoto (1998), a Psicologia é uma profissão formada majoritariamente por mulheres, porém, embora seja possível observar que na composição das bolsas PQ ocorre um crescimento na participação de homens, os determinantes para a alteração desse quadro são desconhecidos.

Em relação ao nível da bolsa, para a amostra geral, observa-se um predomínio da categoria 2 $(58,6 \%)$, seguida da categoria 1D $(13,1 \%)$. Não houve diferença significativa para esses dois níveis de bolsa em função do sexo $(p=0,820)$. Os dados também revelam que a maioria dos pesquisadores com bolsa de produtividade em pesquisa em Psicologia é vinculada a instituições públicas $(79,1 \%)$ - federais $(55,2 \%)$ e estaduais $(23,9 \%)$.

Tabela 3. Caracterização demográfica dos pesquisadores com bolsa PQ

\begin{tabular}{|c|c|c|c|c|}
\hline Características & & & $\mathbf{n}$ & $\%$ \\
\hline Sexo & $\begin{array}{l}\text { Masculino } \\
\text { Feminino }\end{array}$ & & $\begin{array}{l}110 \\
187\end{array}$ & $\begin{array}{l}37,0 \\
63,0\end{array}$ \\
\hline Nível da Bolsa & $\begin{array}{l}\text { PQ-Sênior } \\
\text { PQ-1A } \\
\text { PQ-1B } \\
\text { PQ-1C } \\
\text { PQ-1D } \\
\text { PQ-2 } \\
\text { PQ-2F }\end{array}$ & & $\begin{array}{c}01 \\
23 \\
30 \\
24 \\
39 \\
174 \\
06\end{array}$ & $\begin{array}{c}0,3 \\
7,7 \\
10,1 \\
8,1 \\
13,1 \\
58,6 \\
2,0\end{array}$ \\
\hline \multirow{2}{*}{$\begin{array}{l}\text { Nível da Bolsa } \\
\text { em função do } \\
\text { sexo }\end{array}$} & Masculino & $\begin{array}{l}\text { PQ-Sênior } \\
\text { PQ-1A } \\
\text { PQ-1B } \\
\text { PQ-1C } \\
\text { PQ-1D } \\
\text { PQ-2 } \\
\text { PQ-2F }\end{array}$ & $\begin{array}{l}-- \\
10 \\
11 \\
09 \\
18 \\
60 \\
02\end{array}$ & $\begin{array}{c}0,0 \\
9,1 \\
10,0 \\
8,2 \\
16,4 \\
54,5 \\
1,8\end{array}$ \\
\hline & Feminino & $\begin{array}{l}\text { PQ-Sênior } \\
\text { PQ-1A } \\
\text { PQ-1B } \\
\text { PQ-1C } \\
\text { PQ-1D } \\
\text { PQ-2 } \\
\text { PQ-2F }\end{array}$ & $\begin{array}{c}01 \\
13 \\
19 \\
15 \\
21 \\
114 \\
04\end{array}$ & $\begin{array}{c}0,5 \\
7,0 \\
10,2 \\
8,0 \\
11,2 \\
61,0 \\
2,1\end{array}$ \\
\hline $\begin{array}{l}\text { Categoria } \\
\text { Administrativa } \\
\text { da instituição }\end{array}$ & $\begin{array}{l}\text { Privada } \\
\text { Pública estadual } \\
\text { Pública federal }\end{array}$ & & $\begin{array}{c}62 \\
71 \\
164\end{array}$ & $\begin{array}{l}20,9 \\
23,9 \\
55,2\end{array}$ \\
\hline
\end{tabular}


A Tabela 4 apresenta os dados da produtividade em publicação científica e de orientações dos pesquisadores. Para a amostra geral, os artigos são o tipo de produção cientifica que alcançam escores mais altos $(M=15,91 ; D P=10,68)$, seguidos pelos capítulos de livro $(M=8,29 ; D P=9,53)$. Quanto aos números de orientações, a maior média foi observada para os alunos de mestrado $(M=18,48 ; D P=13,26)$, seguida de alunos de iniciação científica $(M=17,83 ; D P=14,86)$. Há de se considerar, todavia, que a prática de orientação de alunos, seja de graduandos, seja de nível stricto sensu não depende exclusivamente da motivação ou do interesse do docente. Coexistem fatores institucionais, políticos e de fomento científico que estabelecem relações com as atividades de orientação dos pesquisadores (Rodrigues, 1996).

Hutz et al. estimaram, com base no percentual de crescimento da produção no período de 1998 a 2006, que a produtividade dos docentes em programas de pós-graduação em Psicologia no Brasil, no triênio 2007 a 2009, se configuraria da seguinte forma: médias de 2,26 artigos/docente, 1,44 capítulos de livro/docente, 0,17 livros de autoria integral/docente e 0,15 livros organizados/ docente. Comparando essa estimativa com as médias apresentadas pelos pesquisadores com bolsas de produtividade, aparece a superioridade em termos quantitativos de produção desses pesquisadores para a média geral (ver Tabela 4). Embora no presente estudo tenham sido coletadas todas as publicações dos últimos cinco anos, quando controlado o tempo para comparação em produção por triênio, as médias dos índices dos bolsistas PQ foram de 9,55 artigos/docente, 4,97 capítulos de livro/docente e 1,10 livros/docente. Essas médias são de três a quatro vezes maiores que as médias estimadas por Hutz et al. para a população geral dos pesquisadores de programas de pós-graduação em Psicologia no Brasil.

Tabela 4. Produtividade científica da amostra geral e em função do nível da bolsa por grupos

\begin{tabular}{|c|c|c|c|c|c|c|c|}
\hline & \multirow{2}{*}{$\begin{array}{c}\text { Geral } \\
M(D P)\end{array}$} & \multicolumn{2}{|c|}{ PQ SR, 1A e $1 B(n=54)$} & \multicolumn{2}{|c|}{ PQ $1 C$ e $1 D(n=63)$} & \multicolumn{2}{|c|}{ PQ 2 e $2 F(n=180)$} \\
\hline & & $M(D P)$ & Amplitude & $M(D P)$ & Amplitude & $M(D P)$ & Amplitude \\
\hline Artigos & $15,91(10,68)$ & $20,94(12,21)$ & $5-59$ & $18,06(12,24)$ & $4-81$ & $13,64(8,84)$ & $1-50$ \\
\hline Livros & $1,83(3,37)$ & $3,46(6,52)$ & $0-43$ & $1,94(2,50)$ & $0-10$ & $1,30(1,70)$ & $0-10$ \\
\hline Capítulos de livro & $8,29(9,53)$ & $11,85(14,05)$ & $0-102$ & $9,35(9,07)$ & $0-60$ & $6,84(7,54)$ & $0-84$ \\
\hline Iniciação científica & $17,83(14,86)$ & $25,46(18,45)$ & $0-84$ & $22,71(14,44)$ & $0-66$ & $13,83(12,25)$ & $0-61$ \\
\hline Mestrado & $18,48(13,26)$ & $30,43(14,92)$ & $12-84$ & $22,43(11,70)$ & $4-75$ & $13,51(10,19)$ & $0-68$ \\
\hline Doutorado & $5,91(6,84)$ & $12,44(8,47)$ & $0-50$ & $7,56(6,38)$ & $0-30$ & $3,37(4,67)$ & $0-28$ \\
\hline Pós-doutorado & $0,58(1,59)$ & $1,28(1,79)$ & $0-7$ & $0,57(1,45)$ & $0-7$ & $0,37(1,52)$ & $0-17$ \\
\hline
\end{tabular}

Houve casos em que a produção de artigos publicados pelos pesquisadores com bolsas nível 2 e 2F, desde 2007, foi de um único artigo. Houve, também, casos de profissionais que não apresentaram produções de livros ou de capítulos de livro publicados. Tais dados levam à consideração, relativamente contraditória, de que a produtividade dos pesquisadores nesses quesitos não é condição imprescindível para possuir bolsa de produtividade em pesquisa, ao menos em seus níveis iniciais. O mesmo ocorre com as atividades de orientação de estudantes. Alguns pesquisadores com bolsas nível 2 e $2 \mathrm{~F}$ não apresentaram orientações concluídas de estudantes de iniciação científica, mestrado, doutorado ou pós-doutorado. Assim, também o número de orientações concluídas não se mostrou condicionante imprescindível para possuir bolsa de produtividade em pesquisa, ao menos em seus níveis iniciais. A respeito disso, a Resolução Normativa no 009/2009, do Conselho Nacional de Desenvolvimento Científico e Tecnológico, 
corrobora esses achados, uma vez que pontua que, no tocante às bolsas da categoria 2, considera-se a produção científica recente e qualificada, o envolvimento em atividades de editoria científica, as orientações concluídas e os alunos de Iniciação Científica e/ou as monografias de conclusão de curso, de modo que se evidencie estar dando continuidade às investigações iniciadas em seu curso de doutorado (CNPq, 2009).

$\mathrm{Na}$ análise comparativa, para facilitar a interpretação dos dados, os níveis das bolsas de produtividade foram categorizados em três grupos: o primeiro grupo inclui os pesquisadores seniores e os $1 \mathrm{~A}$ e $1 \mathrm{~B}, \mathrm{o}$ segundo, os pesquisadores $1 \mathrm{C}$ e $1 \mathrm{D}$, e o terceiro, com os pesquisadores 2 e $2 \mathrm{~F}$. A Tabela 4 mostra os dados de produtividade dos pesquisadores em função dos três grupos de níveis de bolsa.

Os resultados da ANOVA mostram que houve diferença significativa $(p \leq 0,001)$ entre os grupos para todos os indicadores de produtividade de publicação e de orientação investigados. Como expressa a Tabela 4, para todos os indicadores com diferenças estatisticamente significativas, pesquisadores com bolsas de nível mais alto demonstraram maiores médias sequencialmente (PQ SR-1A1B > PQ 1C-1D > PQ 2-2F).

Testes post-hoc (Bonferroni) demonstraram que, para o número de artigos publicados, os grupos SR-1A-1B e 1C-1D apresentaram médias significativamente maiores que o grupo $2-2 \mathrm{~F}$ ( $p<0,001$ e $p=0,01$, respectivamente). Não foi encontrada diferença significativa entre os dois primeiros grupos $(p=0,397)$. Para o número de livros publicados, o grupo SR-1A-1B demonstrou maiores médias que os grupos 1C-1D e 2-2F ( $p=0,038$ e $p<0,001$, respectivamente). Não houve diferença significativa entre os dois últimos $(p=0,557)$. Para o número de capítulos de livro publicados, o grupo SR-1A-1B apresentou média significativamente maior que o grupo $2-2 \mathrm{~F}(p=0,002)$. O grupo 1C-1D não apresentou diferenças significativas em relação ao primeiro $(p=0,451)$ ou ao último grupo $(p=0,205)$.

A ausência de diferenças significativas entre os grupos de diferentes categorias de bolsas em alguns quesitos pode ser indicativa de que há outras diferenças, de cunho qualitativo, não abarcadas neste estudo. Os dados coletados foram todos indicadores quantitativos de produtividade, o que deve ser considerado, nas interpretações dos resultados, como uma limitação do estudo, uma vez que a análise para concessão das bolsas no Brasil também obedece a critérios qualitativos outros, como a inserção dos proponentes à bolsa dentro da comunidade científica, o mérito técnico-científico e a viabilidade de execução do projeto apresentado. Além disso, a própria produtividade científica é analisada não somente em termos numéricos mas também quanto a outros critérios, como, por exemplo, o extrato Qualis das publicações (CNPq, 2010).

Quanto ao número de orientações de iniciação científica, os grupos SR-1A-1B e 1C1D apresentaram médias significativamente maiores que o grupo 2-2F ( $p<0,001$ em ambos os casos). Não foi encontrada diferença significativa entre os dois primeiros grupos $(p=0,874)$. Para as orientações de mestrado e de doutorado, foram encontradas diferenças significativas entre os três grupos $(p<0,001)$, tendo as médias seguido a hierarquia dos níveis de bolsa (SR-1A$1 \mathrm{~B}>1 \mathrm{C}-1 \mathrm{D}>2-2 \mathrm{~F}$ ). Por fim, para as orientações de pós-doutorado, o grupo SR1A-1B demonstrou maiores médias que os grupos $1 \mathrm{C}-1 \mathrm{D}$ e $2-2 \mathrm{~F}(p=0,045$ e $p=0,001$, respectivamente). Não houve diferença significativa entre os dois últimos $(p=1,000)$. 


\section{Considerações finais}

Embora não se possa afirmar com precisão quais os seus níveis de crescimento, a produção científica em Psicologia vem se expandindo em número nos últimos anos. Diversos fatores são responsáveis por essa expansão, como o maior número de doutores formados no País, a aprovação e a implementação de novos cursos de pósgraduação stricto sensu e o crescimento do número de periódicos na área (BorgesAndrade \& Menandro, 2002; Hutz et al., 2010; Tourinho \& Bastos, 2010). Além desses, a contínua demanda por resultados, nos programas de pós-graduação, evidenciada por uma crescente exigência de publicações por parte do corpo docente, seja na forma de artigos, seja de livros ou de capítulos de livro, revela-se implicada também pela "onipresença de disciplinas de caráter instrumental", conforme Yamamoto (2010, p. 191), que acentuam o foco na formação para a produtividade científica no País. Ainda que algumas agências tenham recentemente estabelecido travas para o produtivismo desenfreado, essa tem sido uma atitude histórica da comunidade científica que ainda se reflete na atualidade (CNPq, 2010).

Os resultados discutidos neste estudo focalizaram indicadores de produtividade dos pesquisadores, em termos de publicações e de orientações. Foi feita uma leitura quantitativa e transversal do estágio atual do desempenho dos atuais bolsistas PQ da área da Psicologia. Para compreender que aspectos são mediadores dessa produtividade, é preciso levar em consideração fatores que não foram abarcados pelo método empregado no presente trabalho, como, por exemplo, a participação em conferências nacionais e internacionais, a criação de redes de colaboração e o treinamento técnico focado no desempenho acadêmico, que são mediadores auxiliares no crescimento das taxas de produtividade dos pesquisadores (Marsh \& Hattie, 2002). Em contrapartida, as principais dificuldades à adequada produção científica envolvem a falta de tempo, elevadas cargas de trabalho, demandas com outras atividades não diretamente relacionadas à pesquisa científica, oportunidades insuficientes para empreender a prática de pesquisa e sentimento de instabilidade no emprego, entre outras (Richard et al., 2009). Tais aspectos devem ser ponderados e contextualizados na leitura dos achados deste estudo.

A seleção das propostas para a concessão de bolsas PQ da área da Psicologia, assim como nas demais áreas, obedece a uma lógica de concorrência, e, por contar com restrita cota de bolsas, faz com que cada Comitê Assessor estabeleça critérios de julgamento, qualitativos e quantitativos (CNPq, 2009, 2010). As avaliações multidimensionais parecem preferíveis à adoção de um único critério quando o assunto é a compreensão dos aspectos que elevam a produtividade de pesquisadores e, por conseguinte, justificam o recebimento de auxílios e benefícios por mérito (Marsh \& Hattie, 2002). No Brasil, a análise para concessão das bolsas segue critérios quantitativos como montante de artigos, livros e capítulos publicados em um dado período, bem como critérios analíticos qualitativos, como a inserção dos proponentes à bolsa dentro da comunidade científica, o mérito técnico-científico e a viabilidade de execução do projeto apresentado (CNPq, 2010).

Uma possível limitação deste estudo advém da qualidade dos dados coletados por meio do Sistema de Currículos Lattes. Não foi objetivo do estudo verificar a veracidade ou a consistência das informações disponibilizadas pelos pesquisadores, como também não se objetivou avaliar possíveis 
diferenças individuais de preenchimento dos currículos. No entanto, considerou-se adequada a utilização da plataforma Lattes como base de dados, porque o currículo dos pesquisadores é um dos elementos levados em consideração para a avaliação ao Conselho Nacional de Desenvolvimento Científico e Tecnológico (CNPq) de bolsas de produtividade em pesquisa.

Para estudos futuros, a análise da produção científica com foco na qualidade dos trabalhos produzidos deve ser privilegiada. Devem ser avaliadas, por exemplo, as áreas temáticas nas quais ocorrem as publicações dos pesquisadores bolsistas de produtividade, os extratos Qualis dos periódicos, a proporção de estudos internacionais, a colaboração com discentes sob orientação ou orientados pelos pesquisadores, a interação com equipes internacionais, e assim por diante. Investigações desses aspectos poderiam oferecer subsídios que enriqueceriam a análise dos perfis dos bolsistas PQ em Psicologia. Além desses fatores, outros dados relevantes como o tempo de doutoramento e a formação inicial do docente (área dos seus cursos de graduação, mestrado e doutorado) devem ser avaliados quanto a sua influência na produção científica dos pesquisadores.
A pertinência da inclusão dessas variáveis se sustenta no fato de que elas são consideradas pelo Conselho Nacional de Desenvolvimento Científico e Tecnológico na avaliação para conceder bolsas de produtividade aos pesquisadores (CNPq, 2010).

As descrições realizadas, bem como as comparações feitas entre as diferentes categorias de bolsistas, permitem refletir sobre os critérios de avaliação da produtividade científica. Estudos futuros podem complementar alguns dados encontrados no presente trabalho, de forma a ampliar a compreensão do perfil dos bolsistas PQ da área da Psicologia. É fundamental primar pelo rigor acadêmico, considerando também o compromisso social dos pesquisadores com a publicação das suas investigações. Assim, os indicadores apresentados neste estudo, bem como as discussões que dele decorreram, devem ser entendidas como um esforço para o desenvolvimento da Psicologia como um campo de produção de conhecimento. Assim, a tônica do texto volta-se mais para a reflexão e o compartilhamento acerca dos fatos do que para tomá-lo meramente como diagnóstico, descolado dos inúmeros fatores que perpassam a prática de pesquisa. 


\section{Guilherme Welter Wendt}

Mestre em Psicologia Clínica pela Universidade do Vale do Rio dos Sinos, Porto Alegre - RS - Brasil.

E-mail: guilhermewwendt@gmail.com

Diogo Araújo DeSousa

Doutorando em Psicologia pela Universidade Federal do Rio Grande do Sul, Porto Alegre - RS - Brasil.

E-mail: diogo.a.sousa@gmail.com

\section{Carolina Saraiva de Macedo Lisboa}

Doutora em Psicologia pela Universidade Federal do Rio Grande do Sul e docente do Programa de Pós-Graduação em Psicologia

e da Faculdade de Psicologia da Pontifícia Universidade Católica do Rio Grande do Sul, Porto Alegre - RS - Brasil.

E-mail: lisboacaro@gmail.com

\section{Silvia Helena Koller}

Doutora em Educação pela Pontifícia Universidade Católica do Rio Grande do Sul e docente da Universidade Federal do Rio

Grande do Sul, Porto Alegre - RS - Brasil.

E-mail: silvia.koller@gmail.com

Endereço para envio de correspondência:

Rua Duque de Caxias, 888-205 Centro Histórico. CEP: 90010-282. Porto Alegre, RS.

Recebido 11/08/2011, 1a Reformulação 10/12/2012, Aprovado 11/04/2013. 


\section{Referências}

Avital, M., \& Collopy, F. (2001). Assessing research performance: Implications for selection and motivation. Sprouts: Working Papers on Information Environments, 1(3), 40-61.

Barata, R. B., \& Goldbaum, M. (2003). Perfil dos pesquisadores com bolsa de produtividade em pesquisa do CNPq da área de saúde coletiva. Cadernos de Saúde Pública, 19(6), 1863-1876.

Borges-Andrade, J. E., \& Menandro, P. R. M. (2002). Pósgraduação em psicologia no Brasil: tendências recentes e algumas reflexões para o futuro. In M. L. Seidl de Moura \& P. R. M. Menandro (Orgs.), 9 Simpósio de Pesquisa e Intercâmbio Científico da Anpepp (pp. 33-36). Rio de Janeiro: Associação Nacional de Pesquisa e Pós-Graduação em Psicologia.

Castro, A. A. F., \& Yamamoto, O. H. (2008). A psicologia como profissão feminina: apontamentos para estudo. Estudos de Psicologia, 3(1), 147-158.

Cavalcanti, A. L., \& Pereira, D. S. A. (2008). Perfil do bolsista de produtividade em pesquisa do Conselho Nacional de Desenvolvimento Científico e Tecnológico (CNPq) na área de Odontologia. Revista Brasileira de Pós-Graduação, 5(9), 67-88.

Conselho Nacional de Desenvolvimento Científico e Tecnológico (CNPq). (2009). Resolução Normativa $n^{\circ}$ 009/2009. Estabelece os critérios de concessão de bolsas individuais no país. Brasília, DF: Autor. Recuperado em 20 de junho, 2011 de http://www.cnpq.br.

Conselho Nacional de Desenvolvimento Científico e Tecnológico (CNPq). (2010). Relatório da Reunião do Comitê Assessor da Área de Psicologia. Brasília, DF: Autor. Recuperado em 23 de junho, 2011 de http://www.cnpq.br.

Conselho Nacional de Desenvolvimento Científico e Tecnológico (CNPq). (2012). Mapa de Investimentos do CNPq. Projetos e Bolsas em Vigência. Brasília, DF: Autor. Recuperado em 9 de dezembro, 2012 de http://www. cnpq.br.

Creamer, E. G. (1998). Accessing faculty publication productivity: Issues of equity: ASHE-ERIC higher education report. Washington, DC: The George Washington University Press.

Emden, C. (1998). Establishing a "track record": Research productivity and nursing academe. Australian Journal of Advanced Nursing, 16(1), 29-33.

Hutz, C. S., Rocha, M. L., Spink, M. J. P., \& Menandro, P. R. M. (2010). Perfil, avaliação e metas de produção intelectual dos programas de pós-graduação em psicologia. Psicologia: Reflexão e Crítica, 23(1), 25-34.

Instituto Brasileiro de Geografia e Estatística (IBGE) (2010). Censo da População Brasileira. Recuperado em 15 de junho, 2011, de http://www.ibge.gov.br .
Lee, S., \& Bozeman, B. (2005). The impact of research collaboration on scientific productivity. Social Studies of Science, 35(5), 673-702.

Mendes, P. H. C., Martelli, D. R., Souza, W. P., Quirino Filho, S., \& Martelli-Júnior, H. (2010). Perfil dos pesquisadores bolsistas de produtividade científica em medicina no CNPq, Brasil. Revista Brasileira de Educação Médica, 34(4), 535-541.

Marsh, H. W., \& Hattie, J. (2002). The relationship between research productivity and teaching effectiveness: Complementarity, antagonistic, or independent constructs? The Journal of Higher Education, 73(5), 603-641.

Ramsden, P. (1994). Describing and explaining research productivity. Higher Education 28(2), 207-226.

Richard, C. M., McGrail, M. R., Jones, R., O'Meara, P., Robinson, A., Burley, M., Ray-Barruel, G. A. (2009). Supporting academic publication: Evaluation of a writing course combined with writers' support group. Nurse Education Today, 29, 516-521.

Rodrigues, M. M. P. (1996). Iniciação científica: o que dizem as agências de fomento? In E. M. Bomfim (Org.), Formações em psicologia: pós-graduação e graduação (Coletâneas da Anpepp no. 8, pp. 103-116). Rio de Janeiro: Associação Nacional de Pesquisa e Pós-Graduação em Psicologia.

Salgado, J. F., \& Páez, D. (2007). La productividad científica y el índice h de Hirchs de la psicología social española: convergencia entre indicadores de productividad y comparación con otras areas. Psicothema, 19(2), 179-189.

Santos, N. C. F., Cândido, L. F. O., \& Kuppens, C. L. (2010). Produtividade em pesquisa do CNPq: análise do perfil dos pesquisadores da Química. Química Nova, 33(2), 489-495.

Sax, L. J., Hagedorn, L. S., Arredondo, M., \& Dicrisi, F. A. (2002). Faculty research productivity: Exploring the role of gender and family-related factors. Research in Higher Education, 43(4), 423-445.

Teodorescu, D. (2000). Correlates of faculty publication productivity: A cross-national analysis. Higher Education $39,201-222$.

Tourinho, E. Z., \& Bastos, A. V. B. (2010). Desafios da pósgraduação em Psicologia no Brasil. Psicologia: Reflexão e Crítica, 23(1), 35-46.

Witte, K., \& Rogge, N. (2010). To publish or not to publish? Scientometrics, 85, 657-680.

Yamamoto, O. H. (2010). Pesquisa na psicologia: reflexões sobre a formação. In XIII Simpósio de Pesquisa e Intercâmbio Científico em Psicologia (pp. 186-195). Fortaleza, CE: Associação Nacional de Pesquisa e Pós-Graduação em Psicologia. 